\title{
Unique Motivation \\ for Using Global Social Network Site in Korea
}

\author{
Hyosun $\mathrm{Kim}^{1}$ and Kwang-Hee $\mathrm{Han}^{2}$ \\ ${ }^{1}$ Graduate Program in Cognitive Science, Yonsei University, 134 Shinchon-dong, \\ Seodaemun-gu, Seoul, South Korea \\ ${ }^{2}$ Department of Psychology, Yonsei University, 134 Shinchon-dong, Seodaemun-gu, \\ Seoul, South Korea \\ cogenghyosun@gmail.com
}

\begin{abstract}
This study is an attempt to connect the distinctive motivation of Korean self-expression [1] with growth of the global social network sites (SNSs) in Korea. To explore the motivation using global SNSs, we conducted in-depth interview. The result of the interviews suggests that Korean expected to look more attractive or charming when they have foreign online friends, thus leading them to seek to make such foreign friends on Facebook, a widely used SNS. In addition, a questionnaire was conducted to analyze the correlation between the offline and online tendencies of Koreans to prefer foreign friends. The results showed that the respondents perceived themselves as more attractive if he (she) had a foreign friend offline and as more attractive if he (she) was a person who had comments from foreign friends on Facebook. The above results implicate that there are various motives for using global SNSs, depending on the cultural backgrounds of the users.
\end{abstract}

Keywords: motivation, global Social Network Site, self-expression, foreign friends, rich-get-rich theory.

\section{Introduction}

While global social network sites (SNSs) such as Facebook are growing in many countries, many studies have shown that the motivation for using them can differ according to different cultural backgrounds. According to data from an internet site that compiles findings and statistics related to Facebook (www.socialbakers.com), the penetration rate of Facebook has increased to 10.2 percent of the online population, implying that nearly 4 million people use this SNS. Facebook have widespread appeal in many parts of the world [2]. However, the growth of global SNSs does not indicate a downward trend of local SNS use. Local SNSs grow with global SNSs together [3] in that both show the importance of an online network. Regarding both local and global SNSs, how different is the motivation for using them?

Many previous studies have investigated the motivation for using SNSs. Before the growth of Facebook, studies were conducted within the framework of each country. In Korea, Jung et al. [1] revealed six motivations for using Cyworld, which is the representative local SNS in Korea, based on what was termed user-and-gratification 
framework. Ahn and Han [4] explored the relationship between SNS use and personality. Recently, several researchers have explored the motivation for using a global SNS, and they have started to analyze the differences among cultures as it relates to the use of a global SNS [3,5]. However, these cross-cultural studies are based on the results of research conducted from a western viewpoint. Hence, they may not be suitable by themselves to show the actual difference among cultures. If the results of studies of motivation for using local SNSs are applied to the study of global SNS, it would be helpful to reveal the true differences as regards other cultures.

Jung et al. [1] suggested six motivations for using the local SNS, Cyworld, in Korea: entertainment, self-expression, professional advancement, passing time, communication with family and friends, and trend-following. Among them, selfexpression seemed to be a unique motivation in Korea. Given that self-expression is a distinctive motive in Korea, the aim of the current study was to connect the distinctive motivation of Korean, self-expression with the growth of the global SNSs in Korea. However, we did not insist on a hypothesis at the outset. Though in-depth interviews, we used a bottom-up approach to reveal the hidden motivations.

\section{In-Depth Interviews}

\subsection{Method}

We conducted in-depth interviews with three Koreans, two Britons and one German. In common, their countries have local (Korea-, UK- and Germany-based) SNSs, but all of them use Facebook more often than they use their local SNS. However, they have different cultures. The characteristic of Koreans can be revealed in the differences between how Koreans and Westerns reflect cultural differences through the answers to the interviews.

Participants. Interviewees were six undergraduate students at Yonsei University in Korea. Three foreign students came from the UK (2) and Germany (1) as exchange students for one year. Korean students participated in exchange for course credit. The foreign students received 30,000won(about €19) cash compensation.

Procedure and Measures. To explore the motivation using local and global SNSs, semi-structured interviews were conducted. They lasted 50 60 min. The interview questions were divided according to three main research topics: (1) Why did the participants start to use Facebook or a local SNSs? (2) Who were their friends on Facebook or the local SNS? (3) Why did the participants continue to use Facebook or the local SNS? The languages used in interviews were Korean and English.

\subsection{Result}

The results of interviews suggest that Korean students expected to look more attractive or charming when they have foreign online-friends (especially western friends). Therefore, they would like to meet foreign friends through Facebook. In contrast, western students did not care whether their friends were foreign or not. This result may have the two reasons: The first is the unique emotional state of Korean, as 
characterized by "being envy" [6]. Koreans feel envious of those whom they want to be. This emotion motivates Korean to approach a 'wannabe'. The second reason is related to the meaning of western culture in Korea. Western culture represents globalization in Korea. For example, many universities in Korea used English slogan to show their international abilities [7]. That is, becoming western means being good at English, being a very capable person, and being more attractive than others in Korea. A foreign person may be one whom a Korean wants to emulate. In addition, "being envy" and becoming western may be combined with the unique motivation of Korean, that being self-expression [1]. Korean would like to express their attractiveness and capability by showing that they have many foreign friends. In sum, Facebook offers the opportunity for them to express their attractiveness online by showing comments written by their foreign friends, thus realizing their 'wannabe' motivations online.

The results of interview were consistent with the rich-get-richer theory [8]. This theory seeks to explain the relationship between personality and social networking use. According to the rich-get-richer theory, personality is expressed online as it is in real life. For instance, a high extroverted person may have a large number of friends on Facebook [9]. The point of this theory is that the offline tendency can extend to the online tendency, which is in good agreement with the results of the interviews. Given that the point of the rich-get-richer theory can be applied to emotion or motivation as well as personality, the distinctive motivation for using a global SNS could begin from Koreans' eager offline traits. To clarify the relationship between offline and online propensities, a questionnaire was used.

\section{Questionnaire}

To examine whether Koreans' unique emotions and motivations expand to the online realm, a questionnaire was used to analyze the correlation between the offline and online tendencies.

\subsection{Method}

Participants. Forty-five participants (twenty five men and twenty women) responded in this questionnaire. The participants were undergraduate students at Yonsei University and participated in exchange for course credit.

Procedure and Measures. The participants completed an online survey. The questionnaire measure was organized in three parts. The first part assessed users' offline tendencies to prefer foreign friends. For example, one question was, "I will look more attractive when I have foreign friends." The second part measured the online propensity for comments written by foreign friends and perception of Facebook compared to local SNSs. For instance, two questions were, "A friend looks more attractive when I see a comment written by foreigners on friend's Facebook page" and "Facebook is more suitable for people my age than a local SNS." The last part concerned demographic information such as gender and the use of SNSs, such as the time spend using them. All questions were rated on a 7-point Likert scale. 


\subsection{Result}

To examine whether Koreans' unique emotion and motivation also existed online, we analyzed the correlation between the preferences of comments written by foreign friends and SNS users eager make to foreign friends. The perceptions of Facebook compared a local SNS were added to the correlation analysis. The results are presented in Table 1. A user evaluated oneself as more attractive if he (she) had a foreign friend offline, and this was the same if a Facebook friend had comments from their foreign friends on their Facebook page. Concerning the relationship with the perception of Facebook, he (she) rated oneself as more attractive or more intelligent when using Facebook compared to a local SNS.

Table 1. Correlation between the preferences of foreigners' comments and eagerness to meet foreigners offline and the perception of Facebook and use of Facebook

\begin{tabular}{lcc}
\hline & $\begin{array}{c}\text { Attractiveness of friends } \\
\text { with foreigners' comments }\end{array}$ \\
\cline { 2 - 3 } & $r$ & $p$ \\
\hline Use of Facebook & -.012 & $\begin{array}{c}\text { Not significant } \\
\text { Not significant }\end{array}$ \\
The total number of friends & -.032 & \\
The number of foreign friends & & $P<.001$ \\
& & $P<.01$ \\
Eagar to meet foreigners offline & .396 & $P<.001$ \\
The person looks more attractive if they have foreign friends. & .706 & Not significant \\
I would like to have foreign friends. & & $P<.001$ \\
I look more attractive if I have foreign friends. & .023 & .545 \\
Perception of Facebook compared to a local SNS & .603 & $P<.001$ \\
Facebook is more suitable for people my age than a local SNS. & \\
A person using Facebook looks more intelligent than those using a local SNSs. & \\
I look more attractive when using Facebook than when I use a local SNS. &
\end{tabular}

\section{Discussion}

According to the result of the in-depth interviews, Korean users of a global SNS appear to consider that foreign online-friends make them look more attractive or charming. This is connected with the unique motivation of self-expression, as revealed by Jung et al. [1]. The motivation to express oneself as very capable or attractive is combined with the representation of western culture. Becoming western means becoming globalized, in the same manner as being good at English is regarded as the index of the possibility to succeed in Korea. In local SNSs, Korean had few opportunities to express this when using a global SNS. This result supports the richget-richer theory [8], which holds that the online propensity reflects the offline trend. To clarify the result of the in-depth interview and explain it as quantitative data, we used the questionnaire. The correlation result supported the result of the in-depth interview result. The more attractive he (she) evaluated oneself if he (she) has a foreign friend offline, the more attractive he (she) also evaluated a person who had the comments of foreign friends on Facebook.

This study shows that the motives for using local SNSs and global SNSs do not originate from a common motive. Although an approach that uses common factors to explain the differences among cultures is important in cross-culture studies, a method 
to reveal the distinctiveness of one particular culture is also meaningful. In addition, the results of this study suggest that there are various motives for using global SNSs depending on the different cultural backgrounds of users. Global SNSs have to consider local motivation for greater business success online.

Acknowledgments. This study has been supported by Yonsei university and Brain Korea 21.

\section{References}

1. Jung, T., Youn, H., McClung, S.: Motivations and self-presentation strategies on Koreanbased "Cyworld" weblog format personal homepages. CyberPsychology and Behavior 10(1), 24-31 (2007)

2. Eldon, E.: ComScore, Quantcast, Compete, Nielsen show a strong December for Facebook traffic in the US (January 22, 2010).

http: / /www.insidefacebook.com/2010/01/22/

comscore-quantcast-compete-show-a-strong-december-

forfacebook-traffic-in-the-us/ (accessed 10.01.10)

3. Kim, Y., Sohn, D., Choi, S.M.: Cultural difference in motivations for using social network sites: A comparative study of American and Korean college students. Computers in Human Behavior 27, 365-372 (2011)

4. Ahn, W., Han, K.: Social Network use and personality. In: Proceeding of Korean Society of Design Science 2010, Wonju, South Korea (2010)

5. Vasaloua, A., Joinsona, A.N., Courvoisier, D.: Cultural differences,experience with social networks and then ature of "true commitment" in Facebook. International Journal of Human-Computer Studies 68, 719-728 (2010)

6. Cha, O.A.: Buroum: An Analysis of Benign Envy in Korea. Korean Journal of Social and Personality Psychology 23(2), 171-189 (2009)

7. Yang, C.Y.: Understanding the Linguistic Use of English Slogans in Korean University Homepages. Language and Linguistics 39, 91-111 (2007)

8. Kraut, R., Lundmark, V., Patterson, M., Kiesler, S., Mukopadhyay, T., Scherlis, W.: Internet paradox: A social technology that reduces social involvement and psychological well-being? American Psychologist 53, 1017-1031 (1998)

9. Amichai-Hamburger, Y., Vinitzky, G.: Social network use and personality. Computers in Human Behavior 26, 1289-1295 (2010) 